\title{
Dosimetry for the Irradiation of Polymers by Soft X-Rays
}

\author{
Dedicated to the Memory of the late Professor Ichiro Sakurada
}

\author{
Hideto SoTOBAYASHI, ${ }^{*}$ Frank-Peter Wolf, ${ }^{* *}$ Wolfram SCHNABEL, ${ }^{* * *}$ \\ Nagwa Abou-SAMRA, ${ }^{*}$ and Frithjof ASMUSSEN* \\ * Fritz-Haber-Institut der Max-Planck-Gesellschaft, \\ Faradayweg 4-6, D-1000 Berlin 33, Germany \\ ** Berliner Elektronenspeicherring-Gesellschaft \\ fuer Synchrotronstrahlung $\mathrm{mbH}, \mathrm{BESSY}$, \\ Lentzeallee 100, D-1000 Berlin 33, Germany \\ *** Hahn-Meitner-Institut Berlin GmbH, \\ Glienicker Strasse 100, D-1000 Berlin 39, Germany
}

(Received February 12, 1987)

\begin{abstract}
The power density of synchrotron radiation emitted by the electron storage ring BESSY $(755 \mathrm{MeV})$ in the soft X-ray range was measured in an exposure chamber used for the irradiation of polymer films with the aid of a calorimeter. The experimental data were compared with data calculated with the aid of the Schwinger equation. According to the emittance of the storage ring the electron beam has a finite vertical and horizontal extension and an angular divergence. The broadening effect can be accounted for by a smearing parameter $s$. Compared with theoretical data obtained on the basis of $s=0$ (point source), the maxima of the experimental data were lower by a few percent, typically: $5.2 \%$ when the beam passed a filter combination $(3.6 \mu \mathrm{m}$ $\mathrm{Al}+7.5 \mu \mathrm{m}$ Kapton). Excellent agreement between experiment and theory was found by assuming $s=0.4$. With respect to "homogeneous" irradiations of polymer films which were scanned continuously up and down through the beam, it was concluded that fairly correct values of the power density were calculated with $s=0$, if the vertical amplitude was larger than $\pm 4 \mathrm{~mm}$ at a distance between radiation source and polymer film of $10.67 \mathrm{~m}$.
\end{abstract}

KEY WORDS Dosimetry / Synchrotron Radiation / Soft X-Rays / Power Density / Schwinger Theory / Calorimeter / Source Emittance / Homogeneous Exposure / Polymer Absorber Foil /

One of the important applications of synchrotron radiation is the transfer of structures in the submicron range by means of $\mathrm{X}$ ray lithography, ${ }^{1-3}$ which uses the soft X-ray part of the white synchrotron light spectrum. This method permits a one-to-one shadow projection of a mask with absorbing structures on a transparent membrane onto a silicon wafer coated with an X-ray sensitive resist. Polymer resists ${ }^{4}$ operate on the basis, that structure and solubility of polymers are changed under the influence of soft X-ray radiation. Main chain scissions or crosslinking are induced by the absorption of ener- getic photons by the polymer resists. The sensitivity of the polymer resist can be influenced by choice and by chemical modification of the polymer material.

The radiation sensitivity is usually measured in terms of the $100 \mathrm{eV}$-yield ( $G$-value) which is equivalent to the number of bonds produced or decomposed per $100 \mathrm{eV}$ of the absorbed energy. Therefore, the radiation dose absorbed by the polymer has to be determined.

In principle, the absorbed dose can be calculated from the incident synchrotron radiation power using known spectral absorption data. ${ }^{5}$ For this calculation detailed knowledge of the 
characteristics of the radiation source and the absorption data of the vacuum windows are needed (unless the irradiation is performed in the ultra high vacuum section of the beam line without using windows). Whereas spectral absorption data can be obtained from published data tables, ${ }^{6}$ the synchrotron radiation characteristics can be calculated according to the Schwinger theory ${ }^{7}$ only on the limited basis of a single electron orbiting in the storage ring. In order to obtain realistic values of the power density, the real size of the electron beam, its angular divergence and the geometrical arrangement of the polymer film during the exposure have to be taken into account. In the present paper various aspects concerning the calculation of the power density of synchrotron radiation emitted from the BESSY storage ring will be considered. Emphasis will be given to a system consisting of several absorber foils (filters) placed in front of a polymer film. In addition, the experimental determination of the power density with the aid of a calorimeter will be reported.

\section{X-RAY EXPOSURE SYSTEM}

White synchrotron light from the electron storage ring BESSY, operated at the electron energy of $755 \mathrm{MeV}$, was filtered by two window foils, W1 $(2-3 \mu \mathrm{m} \mathrm{Si}$ or Al) and W2 $(7.5 \mu \mathrm{m}$ Kapton), before entering the exposure chamber at a distance of approximately $10 \mathrm{~m}$ from the source as shown in Figure 1. The windows separated the ultra high vacuum section of the beam line $\left(<10^{-8} \mathrm{mbar}\right)$ from the exposure chamber ( $\simeq 0.02$ mbar). Several valves and bypasses were installed so that the exposure chamber could be flooded with nitrogen to atmospheric pressure and that the pressure in the section between the windows could be controlled.

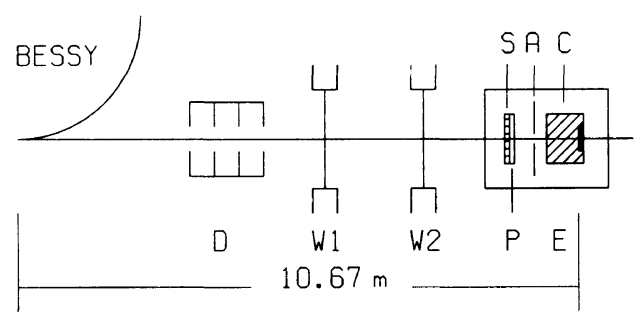

Figure 1. Schematic diagram showing the experimental arrangement at BESSY. D, delay line, aperture $60 \times 10 \mathrm{~mm}^{2}$; W1, Si or Al window foil; .W2, $7.5 \mu \mathrm{m}$ Kapton window foil; E, exposure chamber. A, slit, aperture $4.98 \times 0.55 \mathrm{~mm}^{2}$; $\mathrm{C}$, calorimeter; $\mathrm{P}$, polymer resist; $\mathrm{S}$, substrate foil $(\mathrm{Al})$.

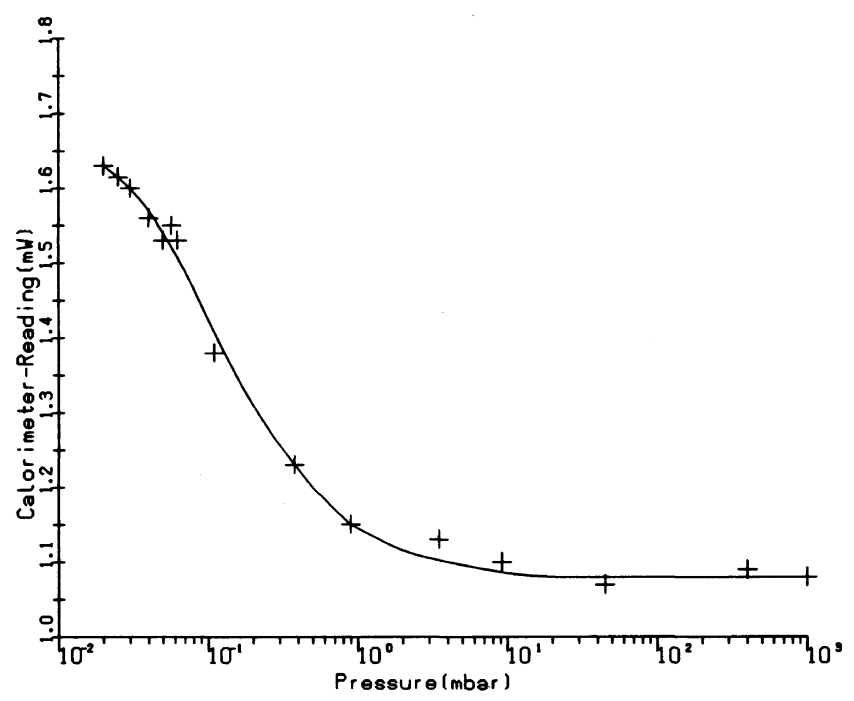

Figure 2. Calorimeter reading as a function of pressure. 


\section{CALORIMETER}

The radiant power of the synchrotron radiation was measured with a calorimeter (Model 154BT MKII, Laser Instrumentation Ltd.) which had been calibrated against a transfer standard (UK National Physical Laboratory) under atmospheric pressure. Because the exposure chamber was operated at approximately $0.02 \mathrm{mbar}$, the reading of the calorimeter as a function of pressure in the range from $10^{3}$ to $10^{-2} \mathrm{mbar}$ had to be measured.

For this purpose the light of a UV lamp, operated at constant voltage, was directed through a quartz glass window flange onto the calorimeter in the exposure chamber. Figure 2 depicts the results. As can be seen from Figure 2 heat transport and convection decrease considerably below $1 \mathrm{mbar}$, so that increasing calorimeter readings are obtained in this pressure range. This curve was used for correcting the calibration of the calorimeter.

The calorimeter was mounted on a drive unit with a pulse counter permitting a variation of its height by $\pm 30 \mathrm{~mm}$. Its vertical position was determined with an accuracy of $\pm 0.02 \mathrm{~mm}$. In order to measure the vertical distribution of the synchrotron radiation a diaphragm of $4.98 \mathrm{~mm}$ horizontal and 0.55 $\mathrm{mm}$ vertical opening was placed at some distance from the active area ( $7 \mathrm{~mm}$ diameter) of the calorimeter, which had a distance of $10.67 \mathrm{~m}$ from the source.

\section{RADIANT POWER FROM A POINT SOURCE}

The Schwinger formula ${ }^{7}$ was used to calculate the spectral power of the radiation $I_{0}$ emitted by a single electron of energy $E$ on a full orbit of radius $R$ under the azimuth angle $\psi$ at the wavelength $\lambda$ :

$$
\begin{aligned}
I_{0}(\lambda, \psi)= & \frac{27}{32 \pi^{3}} \cdot \frac{e^{2} c}{R^{3}} \cdot \frac{\lambda_{c}^{4}}{\lambda^{4}} \cdot \gamma^{8}\left\{1+(\gamma \psi)^{2}\right\}^{2} \\
& \times\left\{K_{2 / 3}^{2}(\xi)+\frac{(\gamma \psi)^{2}}{1+(\gamma \psi)^{2}} K_{1 / 3}^{2}(\xi)\right\}
\end{aligned}
$$

with

$$
\xi=\frac{\lambda_{\mathrm{c}}}{2 \lambda}\left\{1+(\gamma \psi)^{2}\right\}^{3 / 2}
$$

where $\gamma$ is the energy parameter:

$$
\gamma=\frac{E}{m c^{2}}
$$

and $\lambda_{\mathrm{c}}$ is the characteristic wavelength

$$
\lambda_{\mathrm{c}}=\frac{4 \pi R}{3 \gamma^{3}}
$$

$K_{2 / 3}$ and $K_{1 / 3}$ are modified Bessel functions of the second kind. $e$ denotes the charge and $m$ the mass of the electron.

Part of the radiation was absorbed by the wind ow foils according to Lambert-Beer's law:

$$
I=I_{0} \cdot \exp (-\mu d \cdot \rho)
$$

where $I_{0}$ and $I$ denote the spectral power before and after passage of an absorbing layer of thickness $d . \rho$ is the density and $\mu$ the mass absorption coefficient of the material. The mass absorption coefficient $\mu$ depends strongly on the wavelength $\lambda$ of the radiation and the atomic number $Z$ of the absorbing material. Therefore, for a compound consisting of $i$ elements, the resulting mass absorption coefficient at the wavelength $\lambda$ is given by

$$
\mu(\lambda)=\frac{\sum A_{i} \mu_{i}(\lambda)}{\Sigma A_{i}}
$$

with the individual mass absorption coefficients $\mu_{i}(\lambda)$ and the atomic weights $A_{i}$ of the constituents.

For a given angular aperture of the calorimeter, $\Delta \varphi$ in horizontal and $\Delta \psi$ in vertical direction of the calorimeter, the total radiation power $P_{0}$ from a point source is then obtained by integration over $\lambda$ and $\psi$ : 


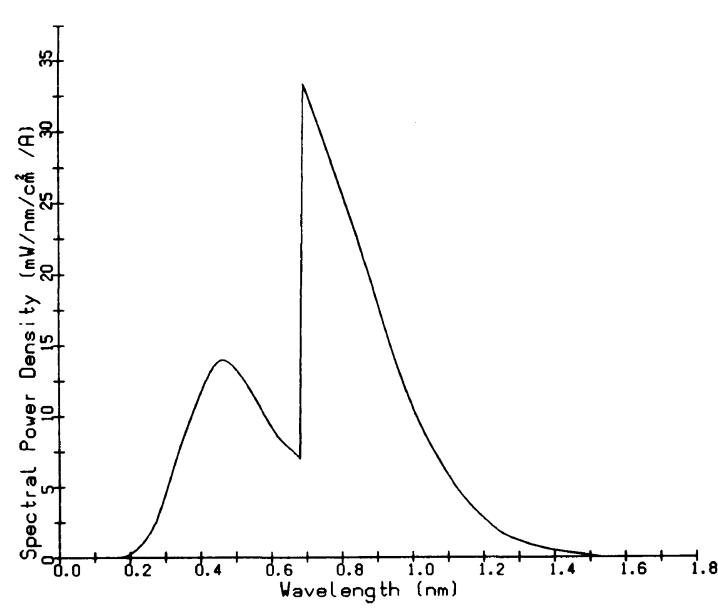

Figure 3. Calculated spectral power density of synchrotron radiation after passage through a couple of window foils ( $2.05 \mu \mathrm{m}$-silicon and $7.5 \mu \mathrm{m}$-Kapton).

$$
P_{0}=\frac{\Delta \varphi}{2 \pi} \iint I(\lambda, \psi) \mathrm{d} \lambda \mathrm{d} \psi
$$

The spectral power density of the synchrotron radiation from BESSY in the exposure chamber (after passing through a $2.05 \mu \mathrm{m}$ silicon and a $7.5 \mu \mathrm{m}$ Kapton foil) has been calculated numerically. ${ }^{8}$ It is plotted in Figure 3 in units of $\mathrm{mW}$ per $\mathrm{cm}^{2}$ per A stored current per nanometer wavelength. This corresponds to an integral over the azimuth angle $\psi$ from -0.47 to $+0.47 \mathrm{mrad}$. The silicon edge can be seen clearly at $\lambda \simeq 0.7 \mathrm{~nm}$.

\section{VERTICAL DISTRIBUTION OF THE RADIATION POWER}

Synchrotron radiation is emitted from the storage ring by a large number of electrons (up to $10^{12}$ at BESSY). These electrons form a beam current $I_{\mathrm{R}}$ according to their charge $e$, number $n$ and time $\tau=2.08 \mathrm{~ns}$ for one full orbit:

$$
I_{\mathrm{R}}=\frac{n \cdot e}{\tau}
$$

The current $I_{\mathrm{R}}$ steadily decreased according to the life time of the stored beam, typically from
$I_{\mathrm{R}}=400 \mathrm{~mA}$ to $150 \mathrm{~mA}$ within $2 \mathrm{~h}$. In order to compare data taken with the calorimeter at different currents $I_{\mathrm{R}}$ they had to be normalized according to $P_{0} / I_{\mathrm{R}}$. The normalized power density quoted in units of $\mathrm{mW} \mathrm{mA}^{-1} \mathrm{~cm}^{-2}$ was obtained by multiplication with the appropriate factor (36.5 for the diaphragm of $4.98 \times 0.55 \mathrm{~mm}^{2}$ ). Whereas the radiation power $P_{0}$ is distributed uniformly in horizontal direction, its distribution in vertical direction has a steep narrow peak in the intersection with the plane of the electron orbit.

This becomes obvious by concidering a typical case, where the synchrotron radiation penetrated two absorber foils $(1.8 \mu \mathrm{m} \mathrm{Si}$ $+7.5 \mu \mathrm{m}$ Kapton). Here, at a vertical shift from the center (plane of the electron orbit) of $\pm 3 \mathrm{~mm}$, the decrease in the power density of the radiation amounted to more than $50 \%$ of the maximum value.

The effects of various absorber foils on the power density of the radiation was studied with three systems. In these cases, the foils were placed perpendicular to the beam in series as indicated in Table I (from left to right).

The normalized radiation power density incident into the opening of the diaphragm of the calorimeter was calculated as a function of the vertical distance from the orbital plane. The calculated data pertaining to a theoretical point source for all three systems were plotted in Figure 4.

System (c) is an example of the arrangement of foils which can be used for the experimental determination of the radiation absorbed in a thin polymer film.

Table I. Combination of absorber foils

\begin{tabular}{|c|c|c|c|c|c|}
\hline \multirow{2}{*}{ System } & \multicolumn{2}{|c|}{$\begin{array}{l}\text { First foil } \\
\text { (W1) }\end{array}$} & \multirow{2}{*}{$\begin{array}{c}\text { Second } \\
\text { foil } \\
\text { (W2) } \\
\text { Kapton }\end{array}$} & \multirow{2}{*}{$\begin{array}{l}\text { Third } \\
\text { foil } \\
\text { (S) } \\
\text { Al }\end{array}$} & \multirow{2}{*}{$\begin{array}{l}\text { Resist } \\
\text { (P) } \\
\text { PMMA }\end{array}$} \\
\hline & $\mathrm{Si}$ & $\mathrm{Al}$ & & & \\
\hline (a) & $1.8 \mu \mathrm{m}$ & - & $7.5 \mu \mathrm{m}$ & - & - \\
\hline (b) & - & $3.6 \mu \mathrm{m}$ & $7.5 \mu \mathrm{m}$ & - & - \\
\hline (c) & - & $3.6 \mu \mathrm{m}$ & $7.5 \mu \mathrm{m}$ & $3.4 \mu \mathrm{m}$ & $3.8 \mu \mathrm{m}$ \\
\hline
\end{tabular}
(W1, W2, S, and P see Figure 1) 


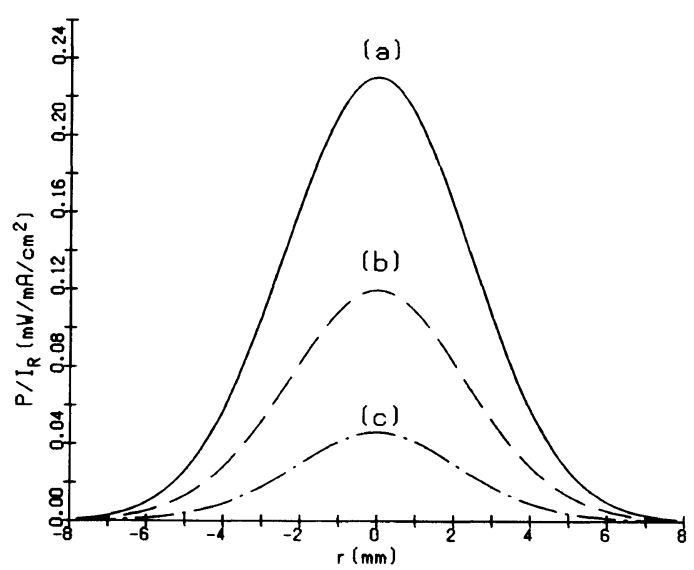

Figure 4. Calculated vertical distribution of the normalized radiation power density pertaining to a point source as a function of the vertical distance $r$ from the orbital plane. Electron energy, $E=755 \mathrm{MeV}$; opening of diaphragm, $4.98 \mathrm{~mm}$ horizontally, $0.55 \mathrm{~mm}$ vertically. Systems (see Table 1): (a) (- - ; (b) (----); (c) (-.-.-).

\section{FINITE SOURCE SIZE AND ANGULAR DIVERGENCE}

According to the emittance of the storage ring, the electron beam has a finite vertical and horizontal extension and angular divergence. The vertical distribution of the electrons in the beam can be approximated by a Gaussian distribution with a full width-half maximum (FWHM) value $\Delta_{y}{ }^{9}$ The angular distribution in azimuthal direction can be characterized by a FWHM value $\Delta_{\psi}$ in a similar way. Both these distributions lead to a broadening of the synchrotron radiation beam and to a broadening of the experimental curves as compared with the calculated curves shown in Figure 4. The horizontal broadening of the beam becomes effective only in the case of narrow horizontal apertures which generate rather broad shadow fringes.

The broadening effect is accounted for by the "smearing" parameter s. $s=0.2$ corresponds to a source size value $\Delta_{y}=0.94 \mathrm{~mm}$ or to an angular divergence $\Delta_{\psi}=0.088 \mathrm{mrad}$. The contributions of these effects add quadratically.
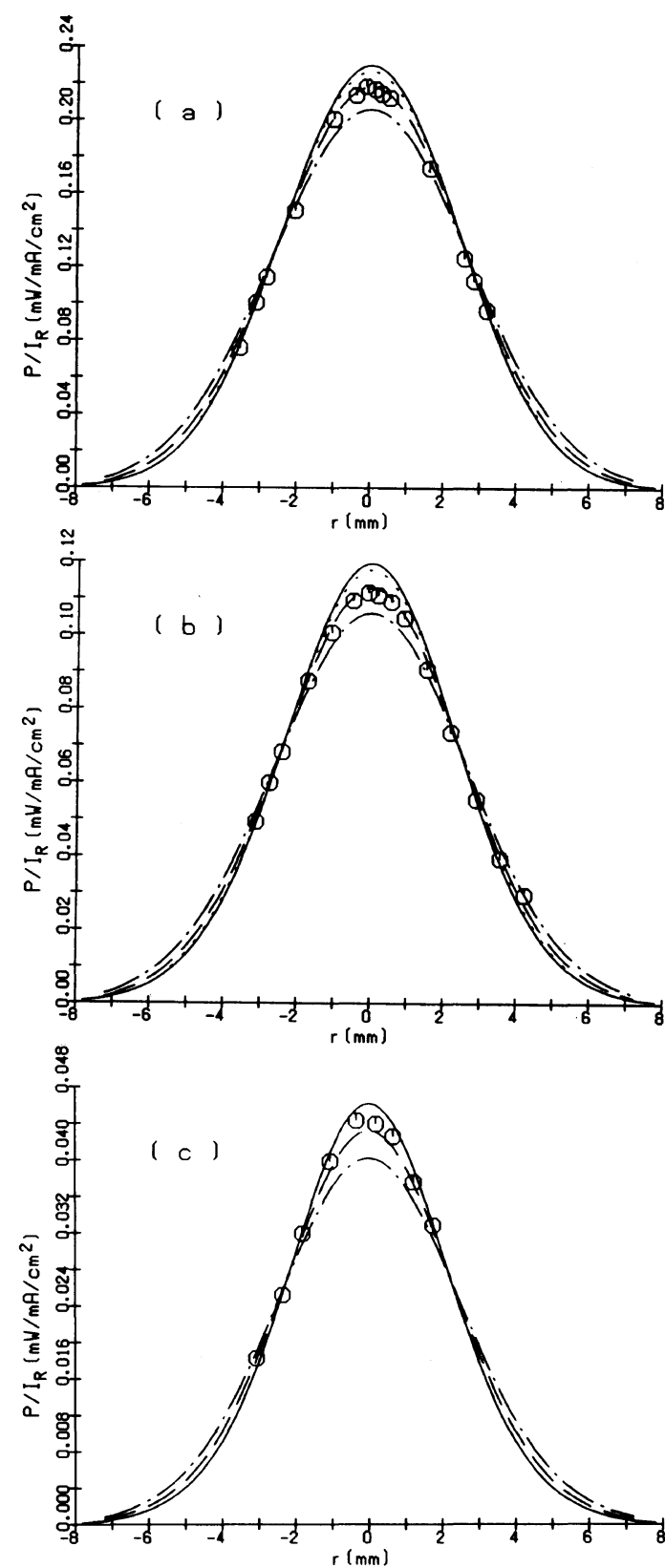

Figure 5. Vertical distribution of normalized radiation power density as a function of the vertical distance $r$ from the orbital plane in three systems (see Table I). Electron energy, $E=755 \mathrm{MeV}$; opening of diaphragm, $4.98 \mathrm{~mm}$ horizontally; $0.55 \mathrm{~mm}$ vertically. Calculated curves: $s=0$ (-); $s=0.2(\cdots) ; s=0.4(----) ; s=0.6$ $(-\cdot \cdot-)$. Experimental values, (0); (a), $1.8 \mu \mathrm{m} \mathrm{Si} \mathrm{and}$ $7.5 \mu \mathrm{m}$ Kapton foils; (b), $3.6 \mu \mathrm{m} \mathrm{Al}$ and $7.5 \mu \mathrm{m}$ Kapton foils; (c), window, $3.6 \mu \mathrm{m}$ and $7.5 \mu \mathrm{m}$ Kapton foils; substrate, $3.4 \mu \mathrm{m} \mathrm{Al}$ foil; polymer resist, $3.8 \mu \mathrm{m}$ PMMA. 
In Figure 5 experimental data of the vertical distribution are compared with data calculated for $s=0,0.2,0.4$, and 0.6 for the systems (a), (b), and (c) as denoted in Table I. As can be seen, the curve calculated with $s=0.4$ yields an excellent fit with the experimental curve for systems (a) and (b). Taking into account that, for system (c), the normalized power density is lower than for system (a) by a factor of 5 , the scatter of the data and the deviation to $s \simeq 0.35$ do not contradict this statement. As compared with data pertaining to a theoretical point source $(s=0)$, the maxima of the experimental data are lower by the following percentages: (a) $-5.2 \%$; (b) $-6.6 \%$; (c) $-4.0 \%$.

Recent measurements of the source size at this white light beam line of BESSY ${ }^{10}$ have shown that the vertical source size is of the order of $\Delta_{y} \leqq 0.2 \mathrm{~mm}$ so that the broadening parameter of $s=0.4$ can almost completely be attributed to the angular divergence $\Delta_{\psi}$ of the electron beam. This value corresponds to a standard deviation of $\sigma_{\psi}=0.075 \mathrm{mrad}$ which is in accordance with the performance data of BESSY. ${ }^{11}$

The source size of the small emittance source of BESSY used at present remains almost constant with decreasing electron current $I_{R},{ }^{10}$ whereas it used to decrease considerably during the life time of each storage ring filling, while BESSY was still operated as a source of larger emittance. ${ }^{9}$ The latter effect had caused a steady increase of the normalized power density of the radiation in the center of the radiation fan (close to the orbital plane) with the consequence of a steady decrease of the broadening parameter $s$.

A plot of the normalized power density incident to the opening of the calorimeter at the position of maximum power $(r \simeq 0)$ during the lifetime of two different fillings of the storage ring is shown in Figure 6. Apart from the values recorded at $I_{\mathrm{R}}>450 \mathrm{~mA}$, where instabilities of the electron beam were observed, all data points could be fitted by a straight line

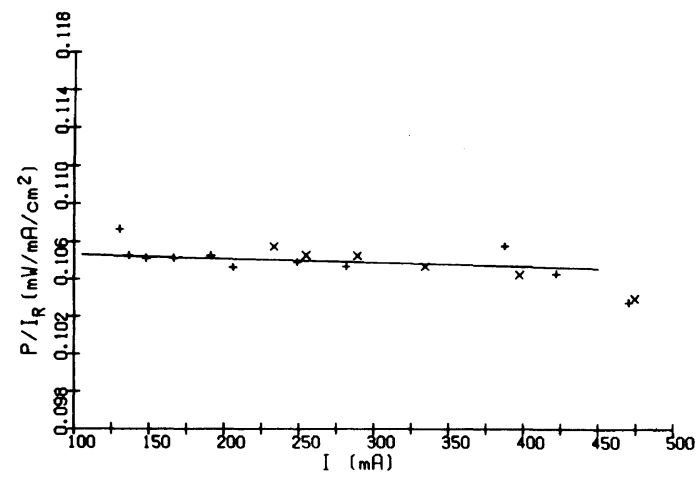

Figure 6. Dependence of the normalized power density at $r=0$ on the storage ring current $I_{\mathrm{R}}$ for two different fillings $(+$ and $x$ ).

exhibiting an increase of $\simeq 0.6 \%$ for $I_{\mathrm{R}}$ decreasing from 400 to $150 \mathrm{~mA}$. This means that a constant value $s=0.4$ can be used for the range $I_{\mathrm{R}} \leqq 400 \mathrm{~mA}$ in order to calculate the radiation power density.

\section{"HOMOGENEOUS" EXPOSURE WITH EXTENDED VERTICAL APERTURE}

In order to perform "homogeneous" exposures of polymer resist samples, the sample holder was moved up and down steadily by a drive unit. Thereby the power density was integrated according to the curves shown in Figure 5 and averaged over the vertical range between the turning points of the drive unit (or between the edges of a diaphragm in the beam line). From Figure 5 it becomes evident that, for a given value of the broadening parameter $s$, the deviation from the integral over the theoretical curve calculated with $s=0$ decreases with increasing vertical aperture $r$.

This deviation, in percent, obtained for system (c) for vertical apertures up to $r=$ $\pm 5.0 \mathrm{~mm}$ and with the parameters $s=0.4$ and 0.6 has been plotted in Figure 7. For a vertical amplitude of $\pm 5 \mathrm{~mm}$ the deviation is reduced to $1 \%$ (for $s=0.4$ ). Figure 7, therefore, represents the limits of accuracy for calculations of the power density using the Schwinger theory without taking into consideration broadening 


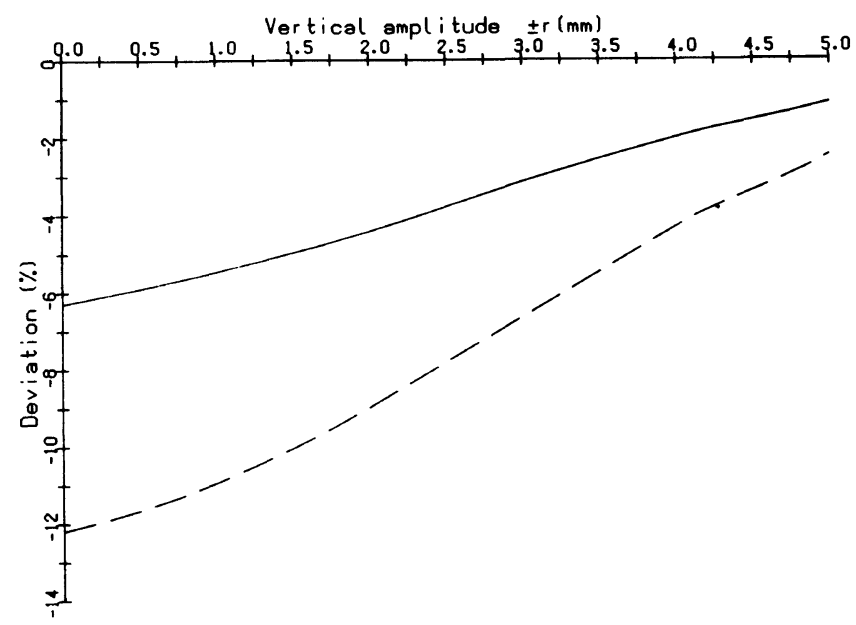

Figure 7. Deviation of the integrated power density at $s=0$ from the integrated power density obtained for $s=0.4(-)$ and $s=0.6(---)$ ) as a function of the vertical amplitude $\pm r$ (evaluated for system (c), see Figure $5(\mathrm{c}))$.

effects caused by the vertical size and the angular dispersion of the source.

The deviations become smaller for larger amplitude values, $\pm r$, of the driving unit. The latter transports the sample vertically through the synchrotron radiation fan. The average power density of the radiation incident to the sample decreases strongly on extending the aperture to higher values. Going from small emittance storage ring optics (small $s$ values) to larger emittance optics, the deviations increase. Provided the angular dispersion $\Delta_{\psi}$ is the predominant contribution to $s$ (as in the case of BESSY, at present), an increase in the distance of the exposure chamber from the source in the storage ring would also enlarge the deviation.

\section{CONCLUSION}

With respect to the irradiation of polymers with the soft X-ray range of synchrotron radiation it was shown that the power density of the radiation at the polymer film can be calculated on the basis of the Schwinger theory which pertains to single orbiting electrons. It turned out that a correction for the broadening of the synchrotron radiation beam due to the finite vertical (and horizontal) extension and the angular divergence of the electron beam is not necessary if an error in the power density of a few percent can be considered negligible. This treatment was found to be applicable for polymer films being scanned through the Xray beam with a vertical amplitude larger than $\pm 4 \mathrm{~mm}$ at a distance between radiation source and polymer film of $10.67 \mathrm{~m}$.

Acknowledgements. Thanks are due to Dr. Oertel, Fraunhofer-Institut fuer Mikrostrukturtechnik, Berlin, for valuable discussions on this paper.

One of the authors (N. Abou-Samra) gratefully thanks Prof. Dr. A. Hamza, Prof. Dr. A. Ghandar of Mansura University, Egypt and Prof. Dr. J. Block of Fritz-Haber Institute of the Max-Planck Society, Berlin West for their supervision and continuous help.

\section{REFERENCES}

1. A. Heuberger, Solid State Technol., Feb. 1986, p 93. 2. H. Betz, F. K. Fey, A. Heuberger, and P. Tischer, 
IEEE Trans Electr. Dev., 26, 693 (1979).

3. K. Hoh, Appl. Phys. (Jpn.), 53, 17 (1984); Extended Abstracts of the 16th International Conference on Solid State Devices and Materials, Kobe, 1984, p 11.

4. W. Schnabel and H. Sotobayashi, Prog. Polym. Sci., 9, 297 (1983).

5. H. Sotobayashi, F. Asmussen, Nagwa Abou-Samra, W. Schnabel, and F. P. Wolf, BESSY Annual Report, 1985, p 254.

6. B. L. Henke, P. Lee, T J. Tanaka, R. L.
Shimabukuro, and B. K. Fujikawa, Atomic Data and Nuclear Data Tables, 27, 1 (1982).

7. J. Schwinger, J. Phys. Rev., 70, 798 (1946); ibid., 75, 1912 (1949).

8. H. Oertel, private communication.

9. F. P. Wolf and W. Peatman, Nucl. Instr. Methods, A246, 408 (1986).

10. F. P. Wolf and P. Kuske, Nucl. Instr. Methods, in press.

11. G. Muelhaupt, BESSY Annual Report, 1985, p 32. 